\title{
Reverse Endoscopic Ultrasound-Guided Gastrojejunostomy for the Treatment of Superior Mesenteric Artery Syndrome: A New Concept
}

\author{
Ming-ming Xu' ${ }^{1}$ Enad Dawod ${ }^{2}$, Monica Gaidhane ${ }^{3}$, Amy Tyberg ${ }^{3}$ and Michel Kahaleh ${ }^{3}$ \\ ${ }^{1}$ Division of Gastroenterology, Kaiser Permanente, Los Angeles, CA, ${ }^{2}$ Division of Gastroenterology, Weill Cornell Medical College, New York, \\ NY, ${ }^{3}$ Division of Gastroenterology and Hepatology, Robert Wood Johnson Medical School, Rutgers University, New Brunswick, NJ, USA
}

Superior mesenteric artery syndrome (SMAS) causes compression and partial or complete obstruction of the duodenum, resulting in abdominal pain, nausea, vomiting, and weight loss. If conservative therapy fails, the patient is typically referred for enteral feeding or laparoscopic gastrojejunostomy.

The last few years have seen increasing use of endoscopic ultrasound-guided gastrojejunostomy (EUS-GJ) for gastric obstruction indications. EUS-GJ involves the creation of a gastric bypass via an echoendoscope in cases in which the small intestine can be punctured under ultrasonographic visualization, resulting in an incision-free, efficient, and safe procedure.

In this case report, we present the first case of SMAS treated using a reverse EUS-GJ, and describe the steps and advantages of the procedure in this particular case. Clin Endosc 2020;53:94-96

Key Words: Endoscopic ultrasound; Gastrojejunostomy; Superior mesenteric artery syndrome

\section{INTRODUCTION}

Palliation of gastric outlet obstruction (GOO) is typically performed with an enteral stent. ${ }^{1}$ Unfortunately, placement of an uncovered metal stent is contraindicated in benign diseases and has been associated with mid-term failure related to intimal hyperplasia. The introduction of lumen-apposing metal stents (LAMSs) has provided an interesting alternative by bypassing the site of obstruction. ${ }^{2}$

Superior mesenteric artery syndrome (SMAS) is a rare disease that induces major malnutrition. When conservative therapy fails, enteral feeding or laparoscopic gastrojejunostomy (LAP-GJ) can be offered to bypass the site of obstruction.

Received: October 30, 2018 Revised: May 8, 2019

Accepted: May 8, 2019

Correspondence: Michel Kahaleh

Division of Gastroenterology and Hepatology, Robert Wood Johnson University Hospital, 1 RWJ Plaza, MEB 464, New Brunswick, NJ 08901, USA

Tel: +1-732-235-7784, Fax: 1-732-235-7784, E-mail: mkahaleh@gmail.com

ORCID: https://orcid.org/0000-0003-0836-6114

(c) This is an Open Access article distributed under the terms of the Creative Commons Attribution Non-Commercial License (http://creativecommons.org/ licenses/by-nc/3.0) which permits unrestricted non-commercial use, distribution, and reproduction in any medium, provided the original work is properly cited.
Recently, several groups have reported on performing endoscopic ultrasound-guided gastrojejunostomy (EUS-GJ) for indications such as GOO.,3 EUS-GJ is a gastrojejunal bypass procedure that involves creating a gastrojejunal fistulous tract using a LAMS under EUS guidance. A direct comparison of EUS-GJ with LAP-GJ for GOO showed similar success rates, although the LAP-GJ group presented with a higher rate of complications. ${ }^{3}$ In this case report, a novel technique is described, which can be offered whenever the small intestine is reached by an echoendoscope.

\section{CASE REPORT}

A 32-year-old woman presented with a 7-month history of progressive abdominal discomfort, post-prandial nausea, poor appetite, emesis, and inability to maintain per-oral diet. She reported unintentional weight loss of $15.9 \mathrm{~kg}$. She weighed 42.4 $\mathrm{kg}$ with a body mass index of $14 \mathrm{~kg} / \mathrm{m}^{2}$ at the time of presentation. The results of laboratory tests (including liver function tests) and an upper endoscopy were unremarkable. However, computed tomography imaging of the abdomen and pelvis showed compression of the second part of the duodenum by 
the superior mesenteric artery, consistent with SMAS (possibly idiopathic). Initial conservative medical treatment with nutritional supplementation and anti-emetics was administered for 3 weeks; however, she continued to lose weight. Consequently, EUS-GJ was offered as an alternative to LAP-GJ for the treatment of SMAS.

EUS-GJ involves the creation of a gastrojejunal bypass under ultrasound guidance. The procedure requires accessing the jejunum from the stomach to create a fistulous tract and then deploying a LAMS across it. ${ }^{3}$ However, for this SMAS case, we used a reverse EUS-guided technique in which the stomach was accessed from the jejunum to create the bypass (Supplementary Video 1).

After 3 days of clear liquid diet, an upper endoscopy was performed, which showed extensive residual fluid and food debris in the stomach that did not clear with suctioning. An extrinsic stenosis was noted in the second part of the duodenum with dilation of the stomach proximal to this obstruction. A nasogastric tube (NGT) was placed for gastric decompression, and the endoscopy was postponed to permit gastric clearance.

After $24 \mathrm{~h}$ of NGT decompression, a linear echoendoscope (TGF-UC180j; Olympus, Center Valley, PA, USA) was inserted into the stomach, which was intentionally filled with water, and then advanced into the jejunum. After the water-filled stomach was identified under EUS guidance, the stomach

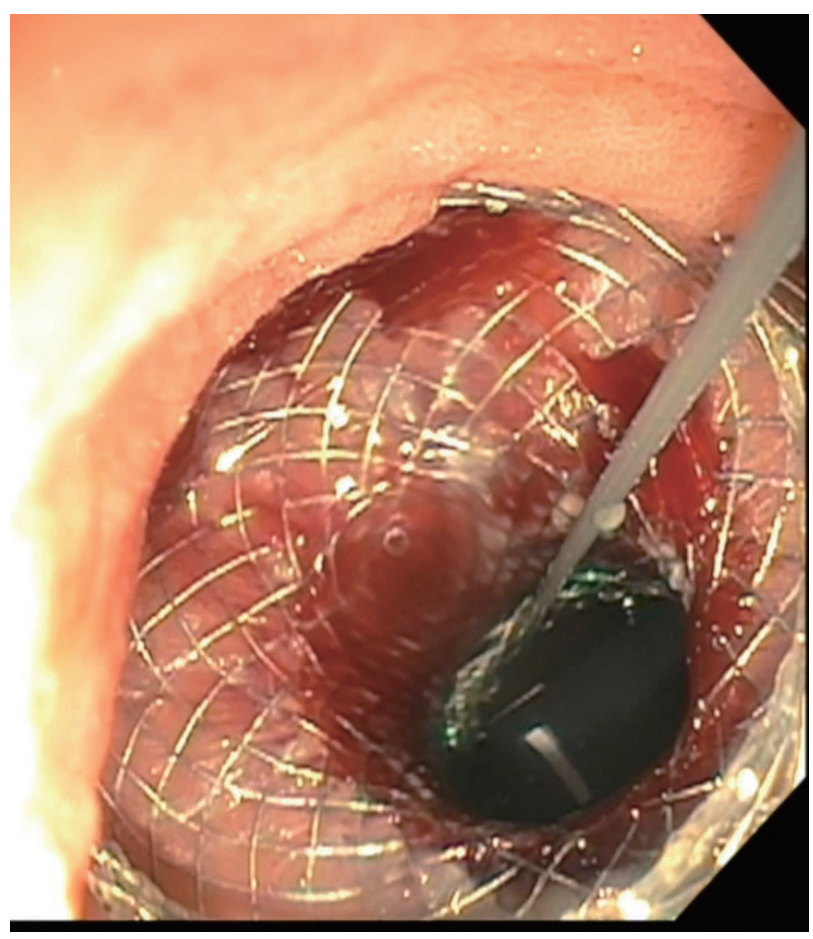

Fig. 1. The lumen-apposing metal stent visualized through the echoendoscope, confirming a successful gastrojejunostomy. was accessed using a 19-gauge fine-needle aspiration needle (Cook Endoscopy, Winston-Salem, NC, USA) to create a fistulous tract. Contrast injection confirmed the access location within the stomach, and a 0.035-in hydrophilic wire was advanced through the needle and coiled within the stomach. A cautery-enhanced 15-mm LAMS (AXIOS; Boston Scientific, Natick, MA, USA) was inserted into the stomach over the wire, and the distal end was deployed under EUS guidance. After pulling the LAMS to appose the stomach wall with the jejunal wall, the proximal end was deployed under endoscopic visualization. A 15-m controlled radial expansion balloon was used to dilate the lumen of the stent to its full diameter. The LAMS was visualized through the echoendoscope, confirming that the distal end of the stent was in the stomach (Fig. 1). A $10 \mathrm{Fr} \times 10 \mathrm{~cm}$ double-pigtail plastic stent (Boston Scientific) was placed across the LAMS (Fig. 2). At 2 months follow-up, the patient reported tolerating a stent diet, resulting in a $4.5 \mathrm{~kg}$ weight gain.

\section{DISCUSSION}

SMAS is characterized by compression of the third portion of the duodenum due to narrowing of the space between the superior mesenteric artery and the aorta, and it has been attributed to loss of the intervening mesenteric fat pad. ${ }^{4}$ Sig-

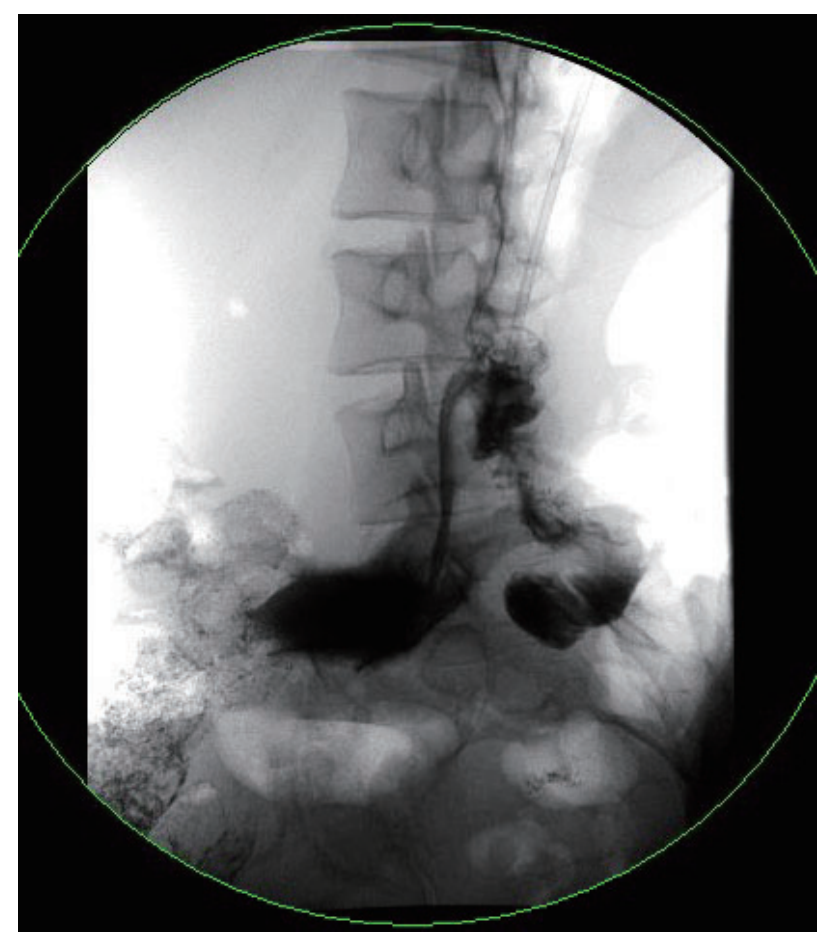

Fig. 2. Fluoroscopic image showing the deployed lumen-apposing metal stent with the contrast transiting through, confirming a functioning gastrojejunostomy. 
nificant weight loss as a consequence of medical disorders, psychological disorders, or surgery is the precipitating factor. ${ }^{5}$

Conservative non-operative therapy is the recommended initial treatment for SMAS. ${ }^{6}$ These conservative measures include NGT decompression, rehydration, electrolyte replacement, and, most important, the use of total parental nutrition or aggressive nutritional support. ${ }^{7,8}$ When conservative management fails, enteral feeding through surgical gastrojejunostomy can be performed. ${ }^{9}$

The advantage of EUS-GJ is that it offers a minimally invasive intervention without the morbidities associated with surgery or the placement of an external feeding tube. It enables preservation of the original anatomy and offers the possibility of procedure reversal without major intervention, by simple removal of the LAMS once the disease has resolved.

This case report shows that a reverse EUS-GJ is a safer and feasible alternative to surgical gastrojejunostomy in patients who fail medical therapy.

\section{Conflicts of Interest}

Michel Kahaleh has received grant support from Boston Scientific, Fujinon, EMcison, Xlumena Inc., W.L. Gore, MaunaKea, Apollo Endosurgery, Cook Endoscopy, ASPIRE Bariatrics, GI Dynamics, NinePoint Medical, Merit Medical, Olympus, and MI Tech. He is a consultant for Boston Scientific, Xlumena Inc., Concordia Laboratories Inc., ABBvie, and MaunaKea Tech. Amy Tyberg is a consultant for Endogastric Solutions. Monica Gaidhane has done consulting work for Interscope Med. She is the COO of Innovative Digestive Health Education \& Research Inc. The other authors have no financial conflicts of interest.

\author{
Author Contributions \\ Conceptualization: Michel Kahaleh \\ Methodology: MK \\ Project administration: Ming-ming Xu, Enad Dawod, Monica Gaidhane \\ Amy Tyberg, MK \\ Resources: MMX, ED, MG, AT, MK
}

Software: MMX, ED, MG, AT, MK

Supervision: MMX, ED, MG, AT, MK

Validation: MMX, ED, MG, AT, MK

Visualization: MK

Writing-original draft: MMX, ED, MG, AT, MK

Writing-review\&editing: MMX, ED, MG, AT, MK

\section{Supplementary Material}

Video 1. Step-by-step video demonstrating the reverse endoscopic ultrasound-guided gastrojejunostomy technique (https://doi.org/10.5946/ ce.2018.196v0001).

\section{REFERENCES}

1. Phillips MS, Gosain S, Bonatti $\mathrm{H}$, et al. Enteral stents for malignancy: a report of 46 consecutive cases over 10 years, with critical review of complications. J Gastrointest Surg 2008;12:2045-2050.

2. Itoi T, Itokawa F, Uraoka T, et al. Novel EUS-guided gastrojejunostomy technique using a new double-balloon enteric tube and lumen-apposing metal stent (with videos). Gastrointest Endosc 2013;78:934-939.

3. Tyberg A, Perez-Miranda M, Sanchez-Ocaña R, et al. Endoscopic ultrasound-guided gastrojejunostomy with a lumen-apposing metal stent: a multicenter, international experience. Endosc Int Open 2016;4:E276-E281.

4. Hines JR, Gore RM, Ballantyne GH. Superior mesenteric artery syndrome. Diagnostic criteria and therapeutic approaches. Am J Surg 1984;148:630-632.

5. Schroeppel TJ, Chilcote WS, Lara MD, Kothari SN. Superior mesenteric artery syndrome after laparoscopic Roux-en-Y gastric bypass. Surgery 2005; 137:383-385.

6. Wang YH, Takada T. Superior mesenteric artery syndrome:--report of four cases. Gastroenterol Jpn 1984;19:479-485.

7. Khodear Y, Al-Ramli W, Bodnar Z. Laparoscopic management of a complicated case of Wilkie's syndrome: a case report. Int J Surg Case Rep 2017;37:177-179.

8. Gersin KS, Heniford BT. Laparoscopic duodenojejunostomy for treatment of superior mesenteric artery syndrome. JSLS 1998;2:281-284.

9. Ylinen P, Kinnunen J, Höckerstedt K. Superior mesenteric artery syndrome. A follow-up study of 16 operated patients. J Clin Gastroenterol 1989;11:386-391. 